Journal of Applied Pharmaceutical Science Vol. 6 (05), pp. 102-111, May, 2016

Available online at http://www.japsonline.com

DOI: $10.7324 / \mathrm{JAPS} .2016 .60516$

ISSN 2231-3354 (cc) EY-NC-SA

\title{
Enhancement of dissolution rate and intestinal stability of candesartan cilexitil
}

\author{
Noha Desouky Fayed*, Mohamed Ali Osman, Gamal Mohammed El Maghraby \\ Department of Pharmaceutical Technology, College of Pharmacy, University of Tanta, Tanta, Egypt.
}

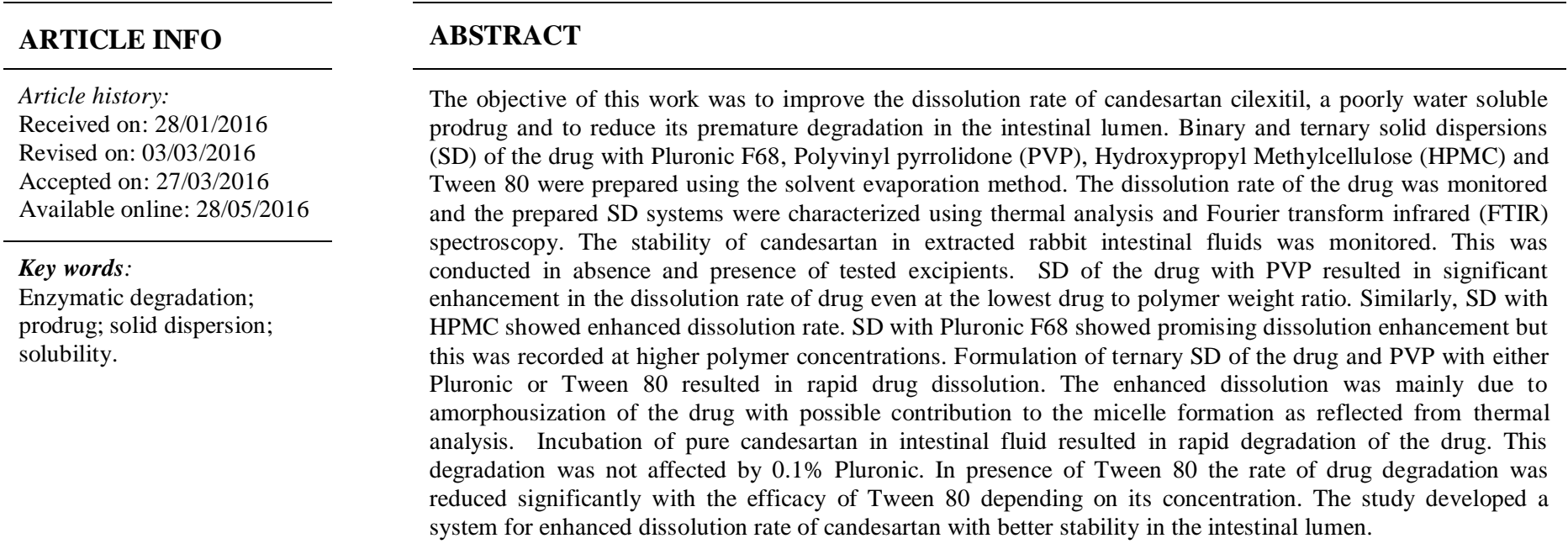

\section{INTRODUCTION}

Candesartan, ( \pm ) $1-\{[($ cyclohexyloxy) carbonyl $]$ oxy $\}$ ethyl 2-ethoxy-1-\{[2'-(1H-tetrazol-5-yl)-biphenyl-4-yl]methyl $\}$ $1 \mathrm{H}$-benzimidazole-7-carboxylate (Figure 1a), is an angiotensin II receptor blocker (ARB) (Detroja et al., 2011). ARBs are widely used in the treatment of diseases like hypertension, heart failure, myocardial infarction and diabetic nephropathy. Candesartan cilexetil performs its action by antagonizing the action of angiotensin II. This can be achieved by blocking the angiotensin type-1 (AT1) receptor. Angiotensin II is the primary vasoactive hormone of the renin angiotensin-aldosterone system with effects

\section{* Corresponding Author}

Noha Desouky Fayed, Department of pharmaceutical technology, College of pharmacy, University of Tanta, Tanta, Egypt

Email:noha_fayed2005@hotmail.com that include vasoconstriction, stimulation of aldosterone secretion, and renal reabsorption of sodium (Husain et al., 2011). Inhibition of such enzyme can have good impact on the blood pressure. Oral administration of candesartan shows low bioavailability, approximately $15 \%$ in humans (Surampalli et al., 2015). Candesartan cilexetil is an ester prodrug of its active metabolite candesartan, which owns its therapeutic effect. The ester form of the drug was developed with the goal of increasing its partition coefficient with subsequent increase in membrane permeability. Accordingly, the prodrug is rapidly and completely bioactivated by carboxylesterase enzyme (ester hydrolysis) after absorption to produce the pharmacologically active form (Husain et al., 2011). Premature degradation of this ester in the intestinal lumen can lead to significant reduction in the membrane permeability to the drug and hence the bioavailability. In vitro investigations revealed instability of the drug in the intestinal fluid. 


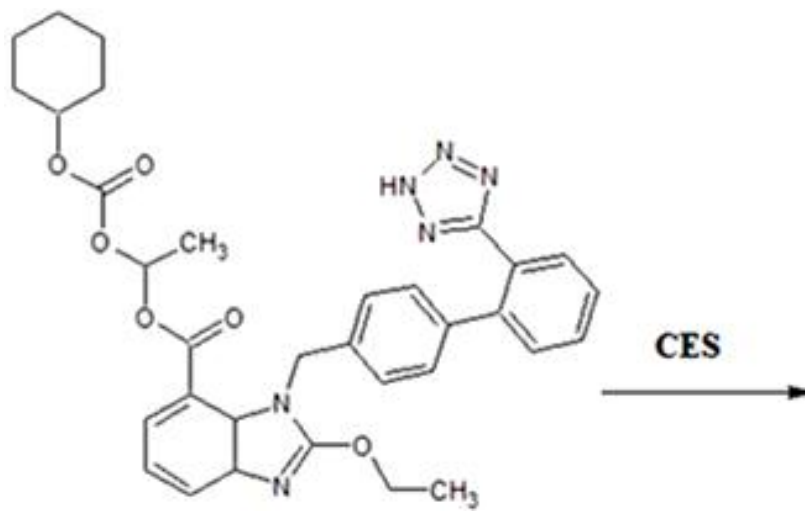

a

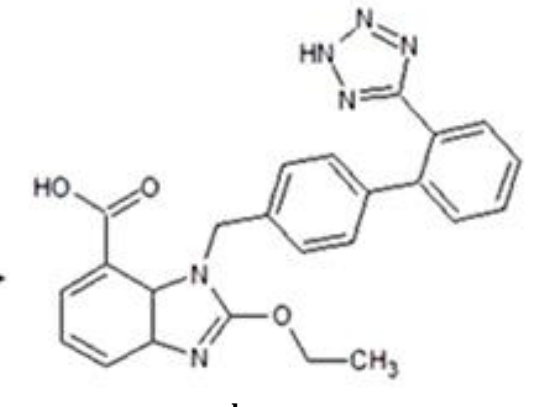

b

Fig. 1: Chemical structure of candesartan cilexetil (a) and its degradation product (b). modified after Borde et al., 2012. CES is Carboxylesterase enzyme.

This was evident both in human and dog intestinal fluids. This degradation was attributed to the enzymatic effect of carboxylesterases 1 and 2 (CES1 and CES2). These esterases are believed to exist in the human intestinal lumen suggesting a role of premature degradation of the prodrug in the reduced oral bioavailability of candesartan (Borde et al., 2012; Laizure et al., 2013). In addition to the premature degradation of the prodrug, its poor dissolution was shown to be another determining factor in reduced oral bioavailability (Surampalli et al., 2015). Alternative strategies were attempted to enhance the dissolution rate and/or bioavailability of candesartan. These included formulation of solid lipid nanoparticles, nanosuspension or nanoemulsion (Nekkanti et al., 2009; Detroja et al., 2011; Gao et al., 2011; Zhang et al., 2012; and Kamalakkannan et al., 2013). Other investigators employed the simple solid dispersion technique for the same purpose (Shaikh and Avachat, 2011; Devi et al., 2014; Gurunath et al., 2014; and Mehta et al., 2014). However, none of these studies considered the effect of these strategies on the premature degradation of the prodrug in the intestinal lumen. Accordingly the objective of this work was to enhance the dissolution rate of the drug and to monitor the effects of dissolution enhancers on the stability of the prodrug in the intestinal lumen. To achieve these goals, solid dispersion technique was adopted using various excipients and rabbit intestinal fluid was used to monitor drug stability.

\section{MATERIALS AND METHODS}

\section{Materials}

Candesartan cilexetil was obtained as gift sample from Pharo Pharma, Borg Elarab, Alexandria, Egypt. Polyvinyl pyrrolidone (PVP) was purchased from Sigma Chemical Co., St Louis USA. Acetonitrile (HPLC grade) was obtained from Fisher Scientific UK, Loughborough, Leics UK. Poloxamer (Pluronic F68), hydroxypropyl methylcellulose E5 (HPMC E5), aerosil R200 (BET surface area $200 \pm 25 \mathrm{~m} 2 / \mathrm{g}$, Loss on drying $\leq 1.5 \%, \mathrm{pH}$ value 3.7-4.5) and Tween 20 were obtained from Sigma pharmaceutical company, Egypt. Tween 80, potassium dihydrogen phosphate, sodium hydroxide, sodium chloride, disodium hydrogen phosphate, potassium chloride and triethylamine (pharmaceutical grade) were purchased from El Nasr Pharmaceutical Chemicals Company, Cairo, Egypt. All drug solutions were freshly prepared before use. Water was filtered through Millipore $0.45 \mu \mathrm{m}$ filter before used.

\section{Chromatography}

Drug analysis utilized high pressure liquid chromatography which employed a chromatographic system from Agilent technologies, 1260 infinity, DE, Germany. The system was comprised a quaternary pump (G1311C) and was equipped with a florescence detector (FLD 1260) and an automatic sampling system (TCC 1260). The whole system was under computer control. Chromatographic separation was performed on a BDS Hypersil C18 reversed phase column of $150 \mathrm{~mm}$ length and 4.6 $\mathrm{mm}$ internal diameter. The average particle size of the stationary phase of the column was $5 \mu \mathrm{m}$ (Thermo scientific, USA). The mobile phase was prepared by mixing $0.02 \mathrm{M}$ of potassium dihydrogen phosphate buffer (adjusted to $\mathrm{pH}$ 6) with acetonitrile and triethylamine at a ratio of 60:40:0.2 (Nekkanti et al., 2010). The mobile phase was pumped isocratically at a rate of $1 \mathrm{ml} / \mathrm{min}$ at ambient temperature. The effluent was monitored by Flourimetric spectrophotometer (excitation wavelengths of $230 \mathrm{~nm}$ and emission wavelength $460 \mathrm{~nm}$ ). The samples were loaded into the HPLC vials before injecting $30 \mu \mathrm{l}$ into the HPLC. Chromatographic data analysis was accomplished using Agilent OpenLAB ChemStation software. The method was validated for linearity, selectivity, precision and lower limit of quantification (LOQ).

\section{Preparation of solid dispersion}

Table 1 presents the composition of the tested solid dispersion formulations. The solid dispersions were prepared by solvent evaporation method. The drug and the polymer were dissolved in a mixture of dichloromethane and ethanol (7:3). This involved adding the drug with stirring until complete solubility. The required amount of polymer was then added to the drug solution and the mixture was stirred to create a clear solution. The solvent was evaporated on a thermosated water bath at a temperature of $50^{\circ} \mathrm{C}$. Aerosil was added gradually after removal of the solvent to produce free flowing mixture. 
Table 1: The compositions of the tested formulations presented as weight ratios.

\begin{tabular}{|c|c|c|c|c|c|c|}
\hline 吾 & 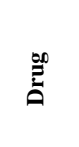 & 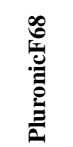 & $\vec{z}$ & $\sum_{\hat{\mid}}^{0}$ & 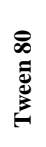 & 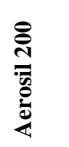 \\
\hline F1 & 33.33 & 33.33 & 0 & 0 & 0 & 33.33 \\
\hline F2 & 20 & 40 & 0 & 0 & 0 & 40 \\
\hline F3 & 11.11 & 44.44 & 0 & 0 & 0 & 44.44 \\
\hline F4 & 33.33 & 0 & 33.33 & 0 & 0 & 33.33 \\
\hline F5 & 25 & 0 & 50 & 0 & 0 & 25 \\
\hline F6 & 40 & 0 & 0 & 40 & 0 & 20 \\
\hline F7 & 25 & 0 & 0 & 50 & 0 & 25 \\
\hline F8 & 25 & 25 & 25 & 0 & 0 & 25 \\
\hline F9 & 25 & 0 & 25 & 0 & 25 & 25 \\
\hline
\end{tabular}

$\mathrm{PVP}=$ Polyvinylpyrrolidone, HPMC $=$ Hydroxy propyl methylcellulose

\section{Determination of drug content and loading efficiency}

Known amount of the solid dispersion formulation was dissolved in methanol with the aid of bath sonication. This was suitably diluted with the dissolution media before determination of the concentration of the drug in solution by HPLC. Experiments were done in triplicate. The drug content was calculated as a percentage of drug relative to the total weight of the formulation. The loading efficiency was calculated as the amount of drug recovered relative to the initial amount of drug added (expressed as percentage).

\section{Characterization of solid dispersions \\ Fourier Transform Infrared (FTIR) spectroscopy}

FTIR studies was conducted to investigate any interaction between the drug and the tested polymers. The studies utilized FTIR spectrophotometer (Bruker Tensor 27, Germany), working in potassium bromide diffuse reflectance mode for collection of IR data. The samples were dry mixed with potassium bromide (spectroscopic grade) before being compressed into disks using hydraulic press. The disks were subjected to FTIR scanning from 4000 to $400 \mathrm{~cm}^{-1}$. Data analysis was performed using Opus IR, FT IR spectroscopy Software.

\section{Differential Thermal Analysis (DTA)}

Thermal analysis of the samples was performed on differential thermal analyzer (Shimadzu, DTA-60H, Japan). Samples of the drug loaded into aluminium pans which were crimped. The loaded samples were subjected to thermal analysis in the temperature range of $30-300^{\circ} \mathrm{C}$ at a heating rate of $10^{\circ} \mathrm{C} / \mathrm{min}$. The thermal behaviour was monitored under nitrogen flowing continuously at a rate of $20 \mathrm{ml} / \mathrm{min}$. The data were collected and analyzed using Simultaneous DTA-TG software.

\section{Dissolution Studies}

The dissolution pattern of the drug was determined using the USP paddle method. The dissolution medium was selected according to the FDA reported dissolution media for candesartan cilexitil dose (32mg). This medium consisted of $0.7 \%$ polysorbate 20 in $0.05 \mathrm{M}$ phosphate buffer adjusted to $\mathrm{pH} 6.5$. The medium was gently transferred into the dissolution vessel so as to minimize foaming of the medium during the experiment. The dissolution medium $(900 \mathrm{ml})$ was equilibrated to and maintained at $37 \pm 0.5^{\circ} \mathrm{C}$ and the paddle speed was adjusted to $50 \mathrm{rpm}$. Dry samples equivalent to $32 \mathrm{mg}$ of pure drug was added to a dissolution vessel. Samples $(5 \mathrm{ml})$ were collected at a predetermined time intervals $(5,10,15,30,45,60$ and $90 \mathrm{~min})$. These were immediately filtered through a $0.45 \mu \mathrm{m}$ Millipore filter and analysed for the drug content by the validated HPLC method. The dissolution medium was replenished with fresh dissolution fluid to maintain a constant volume after each sample. Each dissolution test was performed in triplicate. The dissolution profiles were obtained from the plots of the cumulative amount dissolved as a function of time. These profiles were used to calculate the dissolution parameters which included the amount of drug dissolved in the first 5 minutes (Q5) and the dissolution efficiency (DE). The dissolution efficiency (DE) of a pharmaceutical dosage form is defined as the area under the dissolution curve between time points $\mathrm{t} 1$ and $\mathrm{t} 2$, expressed as a percentage of the area of the rectangle described by $100 \%$ dissolution in the same time, y100. It can be calculated by the following equation:

$$
D E=\frac{\int_{1}^{2} \mathrm{ydt}}{\mathrm{y}_{100} \times(\mathrm{t} 2-\mathrm{t} 1)} \times 100
$$

where $\mathrm{y}$ is the drug percent dissolved at time $\mathrm{t}$ (Khan and Rhodes, 1972; Khan, 1975; and Costa and Lobo, 2001).

\section{In vitro stability study}

The study protocol was approved by the ethical committee, College of Pharmacy, University of Tanta and was conducted according to Principles of Laboratory Animal Care. This study was performed so as to monitor the possibility for premature degradation of the prodrug in the intestinal fluids. To achieve this aim, freshly excised clean segments of rabbit small intestine $(20 \mathrm{~cm}$ each) and colon $(5 \mathrm{~cm}$ each) were inverted. These were subjected to gentle extraction of the mucosal surface. The product of this procedure was dispersed in phosphate buffer saline containing known concentration of the drug which was dissolved alone or in presence of the tested pharmaceutical excipients. These dispersions were incubated in a thermostated water bath maintained at $37^{\circ} \mathrm{C}$. Samples were collected at predetermined time intervals $(0,0.5,1,1.5,2,2.5$ and 3 hours). The samples were immediately filtered through $0.2 \mu \mathrm{m}$ syringe filter before determination of the drug content using the HPLC method. The sample $(30 \mu \mathrm{l})$ was injected without dilution into the previously described HPLC (See before). The percentage concentration of the drug remaining was calculated with reference to the initial drug concentration and was plotted as a function of time.

\section{Statistical analysis}

Data analysis employed Kruskal Wallis test with variation between formulations being compared by Tukey`s multiple comparison. This was performed using SPSS 20. 
Table 2: The amount of drug released after 5 minutes (Q5), the dissolution efficiency, the drug content and drug loading efficiency of candesartan prepared in different formulation.

\begin{tabular}{|c|c|c|c|c|}
\hline Formula & Q5min (\%) & Dissolution efficiency (\%) & Drug Content (\%) & Loading efficiency (\%) \\
\hline Pure drug & $12.7(3.1)$ & $41.8(7)$ & 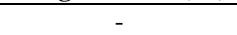 & 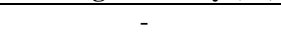 \\
\hline F1 & $17.9(2.1)$ & $50(5.5)$ & 103 (1.4) & 34.33 (1.4) \\
\hline F2 & $21.3(4)$ & $57.6(1.5)$ & $94(2.1)$ & $18.8(2.1)$ \\
\hline F3 & $81.9(2.8)$ & 86.8 (1.5) & $99(0.8)$ & $11(0.8)$ \\
\hline F4 & $76.6(0.2)$ & $82(1.2)$ & $94(2.5)$ & $31.33(2.5)$ \\
\hline F5 & $73.7(0.8)$ & $80.1(2.8)$ & $101(1.1)$ & $25.25(1.1)$ \\
\hline F6 & $50.9(1.8)$ & $74.4(5)$ & $104(3.1)$ & $41.6(3.1)$ \\
\hline F7 & $67.4(1.5)$ & $82.2(0.4)$ & $100(2.6)$ & $25(2.6)$ \\
\hline F8 & $87.6(2.8)$ & $88.3(0.9)$ & $101(2.8)$ & $25.25(2.8)$ \\
\hline F9 & $72(12.1)$ & $83.8(4)$ & $95.3(3.2)$ & $23.83(3.2)$ \\
\hline
\end{tabular}

Values between brackets are S.D. $(n=3)$.

\section{RESULTS AND DISCUSSION}

The technique used in solid dispersion was able to provide homogenous powdered mixtures. The drug loading efficiency in the solid dispersions ranged from $94-104 \%$ of the initial amount added (Table 2). These values exclude any possibility for segregation or degradation of drug during solid dispersion formation. The actual drug content in the formulation was used to take accurate dose of the drug in the dissolution studies (Table 2).

\section{FTIR spectroscopy}

The FTIR spectra of the drug, the polymers and their binary and ternary solid dispersions are shown in Figures 2 and 3. The FTIR spectrum of pure candesartan cilexitil showed the characteristic peaks which were noticed for N-H stretching at 3426 $\mathrm{cm}^{-1}$, for aromatic C-H stretching at $2940 \mathrm{~cm}^{-1}$, for aliphatic C-H stretching at $2862 \mathrm{~cm}^{-1}$, for the two carbonyl groups at 1754 and $1720 \mathrm{~cm}^{-1}$, for $\mathrm{N}-\mathrm{H}$ bending at $1614 \mathrm{~cm}^{-1}$, for the aromatic C-N stretching at $1353 \mathrm{~cm}^{-1}$, for ether C-O stretching at $1036 \mathrm{~cm}^{-1}$ and for the aromatic $\mathrm{C}-\mathrm{H}$ bending at $751 \mathrm{~cm}^{-1}$ (Figure 2). This spectrum correlates with the published spectra for the same drug (Reddy et al., 2012; Gurunath et al., 2014 ;and Surampalli et al., 2015). The FTIR spectrum of pure Pluronic F68 showed the characteristic absorption bands at $3481 \mathrm{~cm}^{-}, 2887 \mathrm{~cm}^{-}{ }^{1}$ and
$1117 \mathrm{~cm}^{-1}$ corresponding to the $\mathrm{OH}$ stretching, C-H stretching and C-O stretching vibrations, respectively (Figure 2). This spectrum correlates with previously recorded one (El Maghraby and Alomrani, 2009). The FTIR spectrum of pure PVP-K30 (Figure 2) showed the characteristic absorption bands at $2955 \mathrm{~cm}^{-1}$ (C-H aliphatic stretching), $1658 \mathrm{~cm}^{-1}$ (C=O stretching), $1495 \mathrm{~cm}^{-1}$ ( $\mathrm{C}=\mathrm{C}$ stretching) and $1288 \mathrm{~cm}^{-1}$ ( $\mathrm{C}-\mathrm{N}$ stretching). This is in good agreement with previous research on the same polymer (Gurunath et al., 2014). The FTIR spectrum of pure HPMC showed the characteristic absorption bands at $3461 \mathrm{~cm}^{-1}$ which corresponds to the $\mathrm{OH}$ stretching. It also revealed the characteristic band for the aliphatic C-H stretching at $2933 \mathrm{~cm}^{-1}$ and that of the aliphatic C-O stretching at $1121 \mathrm{~cm}^{-1}$ (Figure 2). Similar spectrum was recorded by other investigators (El Maghraby and Alomrani, 2009, Sekharan et al., 2011). The FTIR spectrum of pure Tween 80 (Figure 3) showed characteristic peaks at $3449 \mathrm{~cm}^{-1}(\mathrm{OH}$ stretching), $2924 \mathrm{~cm}^{-1}$ (aliphatic $\mathrm{C}-\mathrm{H}$ stretching), $1736 \mathrm{~cm}^{-1}(\mathrm{C}=\mathrm{O}$ stretching), and $1110 \mathrm{~cm}^{-1}$ (aliphatic C-O stretching). This spectrum is similar to that recorded by other investigators (Kura et al., 2014). With respect to the FTIR spectrum of aerosil 200 the spectral features reflected its chemical structure which was revealed as a distinct absorption band at $3448 \mathrm{~cm}^{-1}$ for the $\mathrm{OH}$ group which results from the hydrogen bonding between silica oxygen and water of crystallization or moisture. The absorption band at around $1637 \mathrm{~cm}^{-1}$ is due to $\mathrm{H}-\mathrm{O}-\mathrm{H}$ bending vibration.

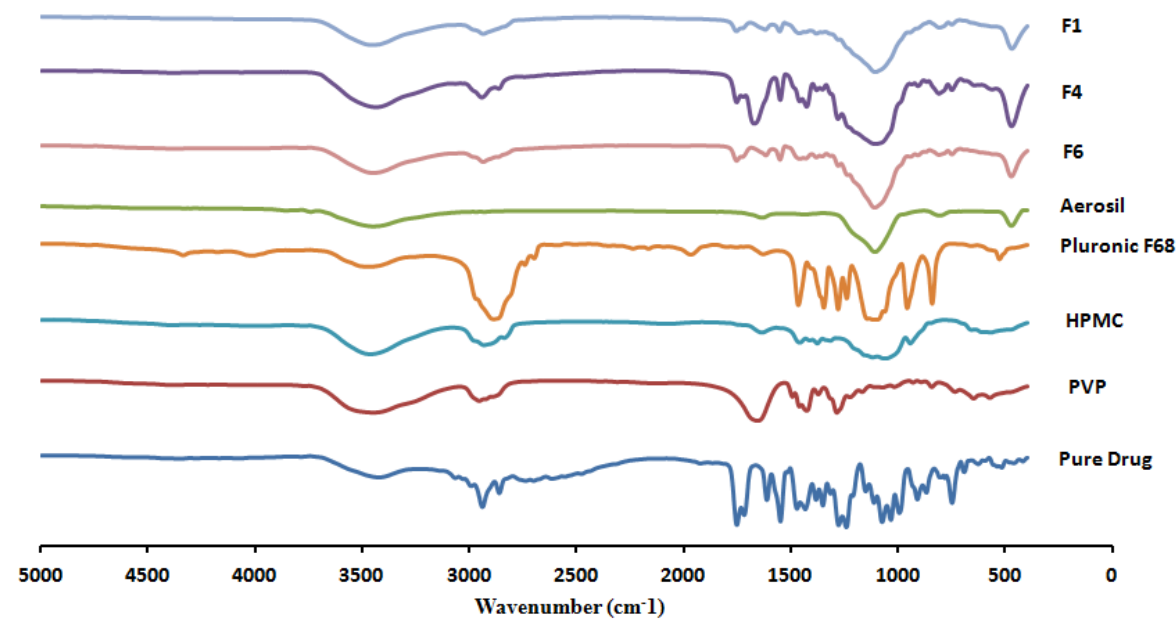

Fig. 2: FTIR spectra of pure drug, polyvinyl pyrrolidine (PVP), hydroxypropyl methylcellulose (HPMC), Pluronic F68, aerosil 200 and their binary solid dispersions. Formulation details are in Table 1. 


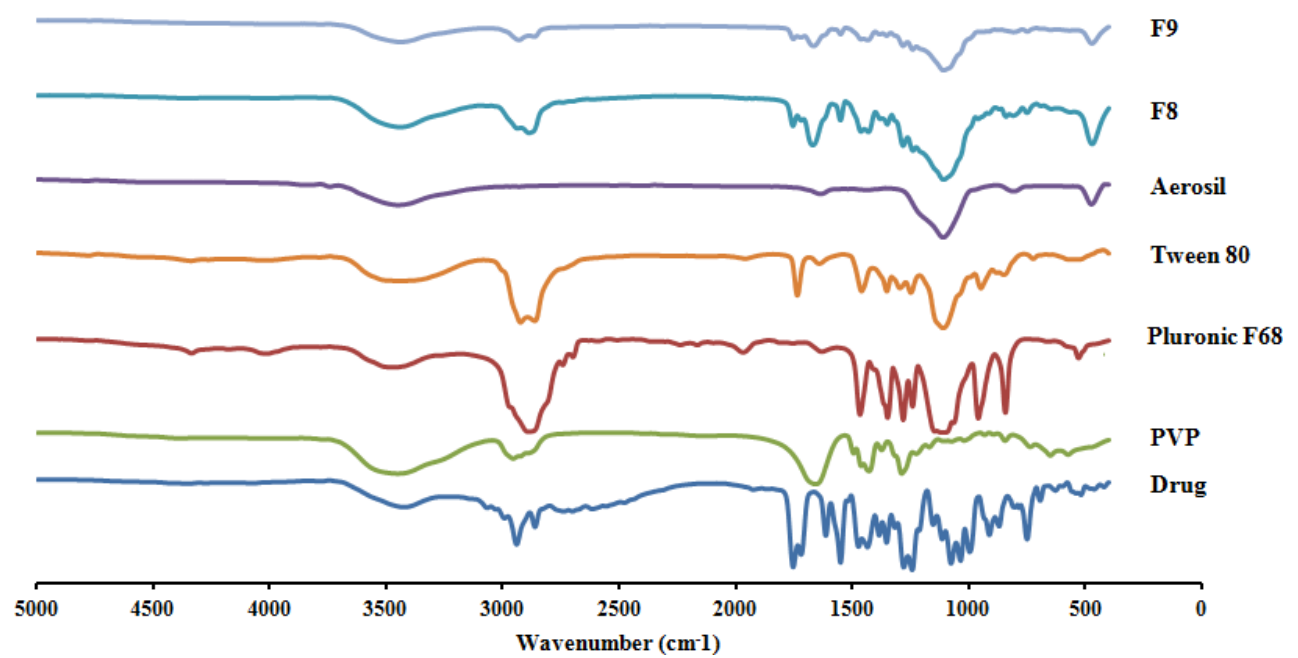

Fig. 3: FTIR spectra of pure drug, polyvinyl pyrrolidine (PVP), Pluronic F68, Tween 80, aerosil \& their ternary solid dispersions. Formulation details are in Table 1.

The $\mathrm{SiO}$ symmetric stretching vibration, the asymmetric $\mathrm{SiO}$ stretching and $\mathrm{SiO}$ bending vibrations were recorded at 1110, 810 and $474 \mathrm{~cm}^{-1}$, respectively (Figures 2 and 3). These features are in good agreement with the published spectrum for the same material (El-Gizawy et al., 2015). The FTIR spectra of binary and ternary solid dispersions reflect spectral features similar to the sum of the spectra of the components of the solid dispersion. This eliminates the possibility of interaction between the drug and the tested excipients (Figures 2 and 3).

\section{Differential thermal analysis (DTA)}

The thermal behavior of the drug, the polymers and their binary and ternary solid dispersions was monitored using DTA. Figure 4 shows examples of the DTA traces. The DTA trace of pure drug showed a sharp endothermic peak at $171.49^{\circ} \mathrm{C}$ with an enthalpy of $4.36 \mathrm{~J} / \mathrm{gm}$. This peak is due to the melting of the drug indicating that the drug is highly crystalline. This endotherm is followed by an exothermic peak at $186.88^{\circ} \mathrm{C}$ which can be attributed to the thermal decomposition of the drug. The recorded thermal behavior of the unprocessed drug is comparable with the literature findings (Nekkanti et al., 2009; Detroja et al., 2011; Gurunath et al., 2014; and Sezgin-Bayindir et al., 2015).

The thermogram of pure Pluronic F68 reflected its melting transition which was recorded as single sharp endothermic peak at $60.21^{\circ} \mathrm{C}$ (Figure $4 \mathrm{a}$ ). This thermogram is similar to the previously reported for the same material (Fathy and El-Badry, 2003; El Maghraby and Alomrani, 2009; and He et al., 2011).

The thermogram of pure PVP showed a very broad endothermic peak in the temperature range of $31.5-129.8^{\circ} \mathrm{C}$. This broad endotherm can be attributed to the evaporation of water and is characteristic for the amorphous PVP (Figure 4b). Similar thermogram was recorded for PVP (Marin et al., 2002; Gurunath et al., 2014; Liu et al., 2014; and Gorajana et al., 2015).

Pure HPMC showed a broad endothermic peak was noticed. This peak has an onset of $32.2^{\circ} \mathrm{C}$ and endset of $128.06^{\circ} \mathrm{C}$ (Figure 4c). This endotherm reflects the release of the adsorbed moisture. Similar thermal pattern was recorded by other investigators and was similarly explained (Barakat et al., 2008; El Maghraby and Alomrani, 2009; and Mesnukul et al., 2009).

For pure aerosil, the thermogram did not show any endothermic peaks which is expected with such amorphous material (Figure 4a). Similar findings were found by other investigators (El-Gizawy et al., 2015).

Formulation of the drug as binary solid dispersion with Pluronic F68 resulted in a significant reduction in the Tm of the melting transition of the drug with significant reduction in the enthalpy of the transition. This effect was noticed at lower ratios of the polymer with the endothermic peak disappearing completely in case of 1:4 weight ratio of the drug to Pluronic F68 (Figure 4a). This effect can be attributed to the formation of amorphous structure of the drug, solubility of the drug in melted polymer which melts at low melting point or formation of eutectic mixture. It should be noted that the recorded thermal behavior of the drug in solid dispersion was associated with broadening of the endothermic peak of Pluronic F68 reflecting reduction of cooperativity of the transition and indicating the dispersion of drug molecules within the matrix structure of the polymer. Similar thermal changes were recorded for the solid dispersion of Pluronic F68 with other drugs (El Maghraby and Alomrani, 2009).

Preparation of the drug in the form of binary solid dispersions with different weight ratios of PVP abolished the main endothermic peak of the drug. This effect was recorded with all of the tested solid dispersion systems (Figure $4 \mathrm{~b}$ ). This suggests possible transformation of the drug from crystalline to amorphous form. It is important to highlight the existence of an exothermic peak at about $247^{\circ} \mathrm{C}$. This peak strengthens with increasing the weight ratio of PVP. This exotherm can be attributed to the decomposition of the solid dispersion system. Amorphousization of candesartan was recorded after solid dispersion formation with PVP (Gurunath et al., 2014). 

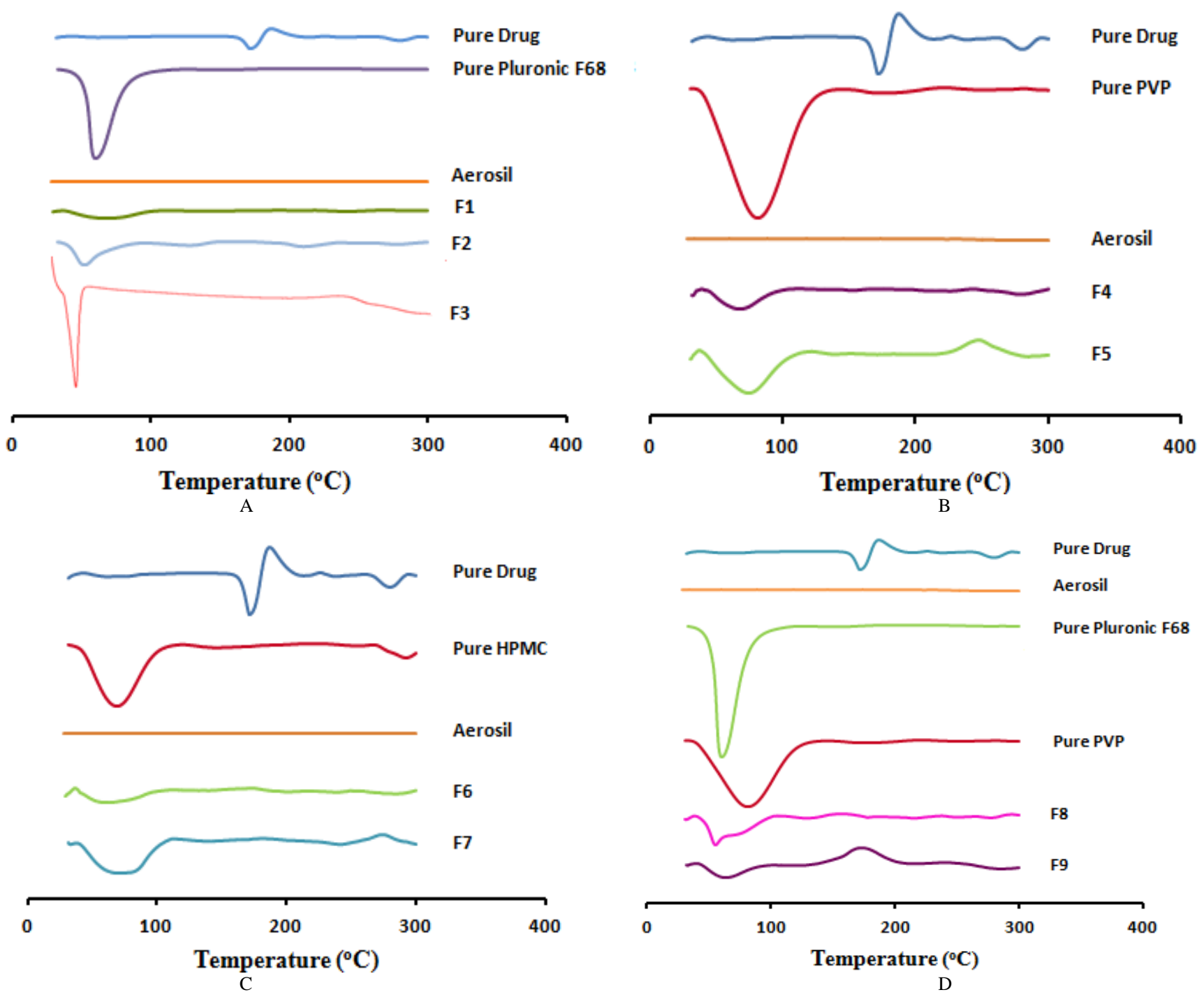

Fig. 4: DTA traces of pure candesartan and its binary and ternary solid dispersions with different polymers. Formulation details are in Table 1.

The thermal behaviour of the drug after formation of binary solid dispersion with HPMC was similar to that recorded in case of solid dispersions with PVP (Figure 4c). This again suggests transformation of the drug into amorphous structure with subsequent decomposition of the solid dispersion at high temperature value. Solid dispersions of HPMC with other lipohpilic drugs showed the same pattern (El Maghraby and Elsergany, 2014).

The prepared ternary solid dispersions in presence of PVP with either Pluronic F68 or Tween 80 also revealed the transformation of the crystalline pure drug into the amorphous form where the characteristic endothermic peak of the pure drug disappeared (Figure 4d). In the Tween 80 containing system, an exotherm was recorded at $173^{\circ} \mathrm{C}$ which can be attributed to the decomposition of the components of the ternary system.

\section{Dissolution studies}

The dissolution profiles of the drug and the prepared solid dispersions are shown in Figure 5. The calculated dissolution parameters are presented in Table 2. The dissolution profile of the pure unprocessed drug revealed erratic and slow dissolution (Figure 5). The drug liberated only $13 \%$ of the dose in the first 5 minutes. The total amount of drug dissolved in 90 minutes was just over $55 \%$. The calculated DE was only $41.8 \%$ (Table 2 ). This poor dissolution behaviour reflects the hydrophobicity of the drug. The dissolution pattern was recorded by other investigators (Devi et al., 2014). Preparation of binary solid dispersions of the drug with Pluronic F68 increased the dissolution rate of the drug but the effect was noticed at the highest tested ratio of the polymer (1:4; drug/Pluronic F68) (F3) (Figure 5).

At this weight ratio the solid dispersion resulted in significant increase in the dissolution parameters $(\mathrm{P}<0.05)$. It was able to liberate $81.9 \%$ of the labelled drug in the first 5 minutes with the overall dissolution efficiency reaching $86.8 \%$. The increase in the dissolution rate can be due to the wetting effect of the polymer which was indicated from rapid dispersion of the dry formula in the dissolution medium which differs from the unprocessed drug which tends to float due to its hydrophobicity. 

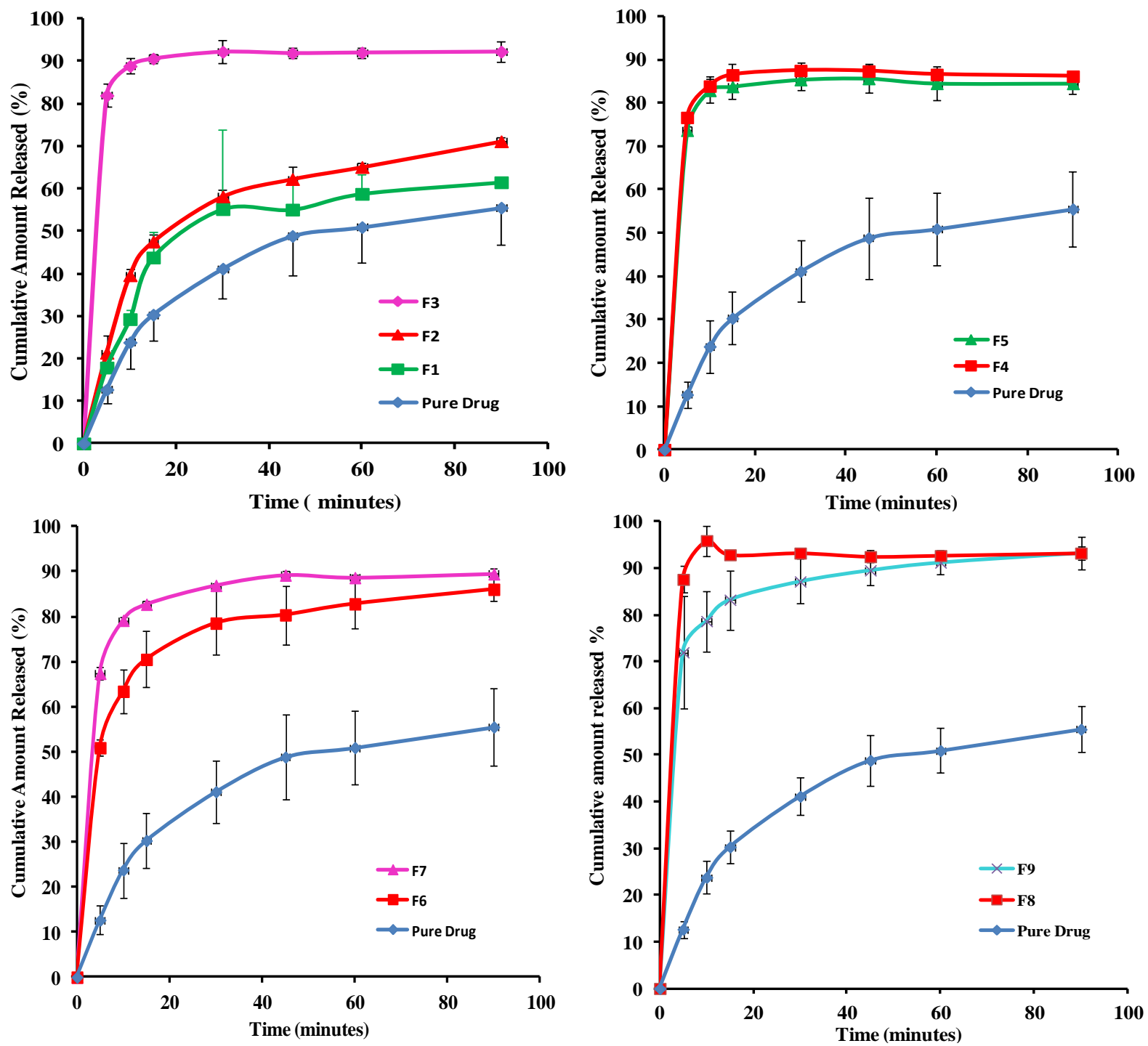

Fig. 5: The dissolution profiles of candesartan in pure state, binary and ternary solid dispersion with different polymers. Formulation details are in Table 1.

Another possible explanation for the enhanced dissolution after solid dispersion formation with Pluoronic F68 is modification of the crystalline structure of the drug as revealed from the thermal analysis results. The ability of Pluronic F68 to self-aggregate forming micelles and liquid crystalline phases was suggested as another mechanism for enhanced dissolution in these systems (Devi et al., 2014). Successful dissolution enhancement was recorded for different lipophilic drugs after solid dispersion formation with Pluronic F68 (El Maghraby and Alomrani, 2009; El Maghraby and Elsergany, 2014; Emami et al., 2014; and Yan et al., 2014).

Formulation of binary solid dispersions of the drug with PVP K30 resulted in significant increase in the dissolution rate of the drug compared to the unprocessed powder (Figure 5). This enhancement in the dissolution rate was shown with the lowest tested concentration of PVP (at 1:1 weight ratio with drug) (F4). Further increase in the ratio of PVP did not result in significant change in the drug release pattern (Figure 5). The amount of the drug dissolved in the first 5 minutes (Q5) was $76.6 \%$ and $73.7 \%$ for solid dispersions containing the drug with PVP at weight ratios of 1:1 and 1:2, respectively (Table 2). The calculated dissolution efficiency values were $82 \%$ and $80.1 \%$ for the same systems, respectively. This indicates that PVP is effective even at the lowest tested concentration. The mechanism of enhanced dissolution after solid dispersion formation of the drug with PVP can be mainly attributed to the change in the crystalline structure of the drug which was evident from the thermal analysis data which reflected the amorphousization of the drug in presence of PVP. The wetting effect of PVP was also suggested by other investigators (Karavas et al., 2007; Emami et al., 2014; Ha et al., 2014; and Meka et al., 2015). Finally, in case of HPMC the binary solid dispersions resulted in significant increase in the dissolution rate with effect starting to be significant at 1:1 (drug: polymer, weight ratio) (F6) (Figure 5). At this ratio the formulation liberated almost 50\% of 
the drug in the first 5 minutes with a DE of $74.4 \%$ (Table 2). The dissolution rate increased with increasing polymer concentration with more than $64 \%$ of the drug being released in the first 5 minutes in case of solid dispersion containing the drug with the polymer at 1:2 weight ratio (F7) with the overall dissolution efficiency reaching $82.2 \%$ (Table 2). The mechanism of improvement in the dissolution parameters of the drug after solid dispersion formation with HPMC can be due to the formation of amorphous structure of the drug. This is confirmed from the thermal analysis results. The wetting effect of the polymer can be considered as another mechanism for enhanced dissolution as indicated from rapid dispersion of the powdered formulation. Binary solid dispersions of other poorly water soluble drugs with HPMC was previously prepared and showed an increase in the dissolution profile of the tested drugs (Boghra et al., 2011; Ha et al., 2015).

Comparing the tested polymers with respect to enhancing drug dissolution, PVP was the most efficient. This conclusion was based on the fact that PVP produced the greatest enhancement in drug dissolution at the lowest weight ratio with the drug compared with other polymers at the same weight ratio. Taking into consideration that the mechanism of enhanced dissolution after solid dispersion with PVP is mainly based on the change in the crystalline structure of the drug, it was decided to explore ternary systems of the drug with PVP and micelle forming materials. This can employ dual mechanisms for dissolution enhancement while maintaining the potential enzymatic inhibitory function of the micelle forming material. Thus ternary solid dispersions were prepared using the drug with PVP and Pluronic F68 or Tween 80. With respect to the ternary system containing Pluronic F68 the recorded dissolution profile showed rapid dissolution pattern with the dispersion liberating more than $87 \%$ of the labelled drug (Figure 5). The overall dissolution efficiency of this ternary system was $88.3 \%$ (Table 2). Replacing Pluronic F68 with Tween 80 produced a ternary system which was able to liberate $72 \%$ of the drug in the first 5 minutes with the overall dissolution efficiency reaching $83.8 \%$ (Figure 5 and Table 2). These results reflected the potential of ternary system formation for enhanced dissolution. Other investigators recorded an increase in the dissolution rate of a poorly soluble drug using a ternary system containing the drug with PVP K30 and Pluronic F68 (Ha et al., 2014). Tween 80 was used in a ternary solid dispersion system by Akbari to enhance dissolution of spironolactone (Akbari et al., 2015). Synergistic dissolution enhancement was recorded after preparation of ternary solid dispersion system of other poorly water soluble drugs (Rashid et al., 2015; Yan et al., 2014).

\section{Effect of micelle forming material on the enzymatic stability of Candesartan}

To probe the possibility of premature degradation of the prodrug in the intestinal lumen, the drug stability was assessed in the fluids extracted from rabbit intestine. The stability was monitored both in presence and absence of the selected excipients. Incubation of pure drug solution in the small intestinal fluids resulted in rapid degradation of the drug with more than $82 \%$ of the drug being degraded in small intestinal fluids after 30 minutes. The same pattern was shown in the colonic fluids in which the drug lost more than $87 \%$ of its potency in the first 30 minutes (Figure 6).
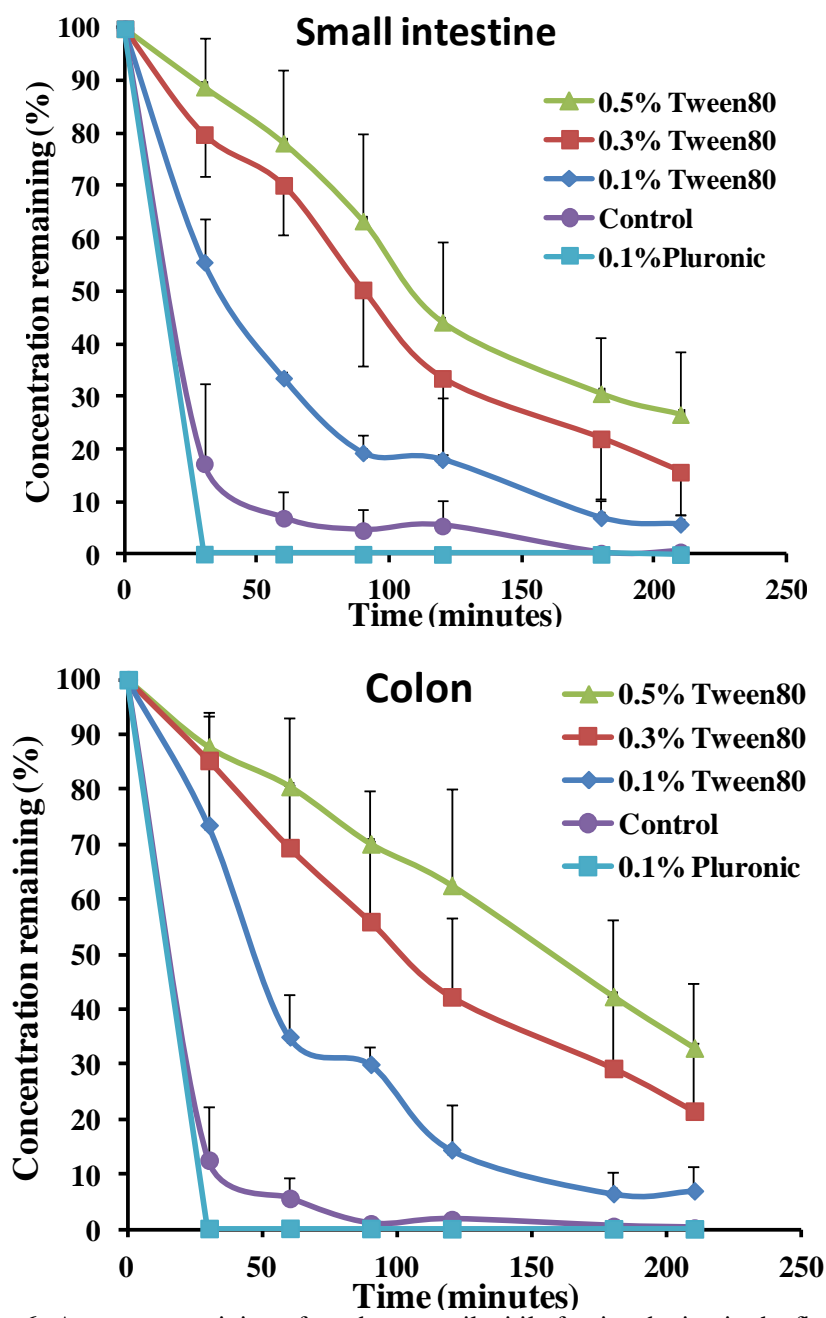

Fig. 6: Amounts remaining of candesartan cilexitil after incubation in the fluids extracted from rabbit intestine both in absence (control) and presence of Pluronic F68 or Tween 80.

These results highlight the existence of premature degradation of the prodrug in the intestinal lumen. Similar presystemic intestinal hydrolysis was recorded for candesartan cilexitil by other investigators (Nishimuta et al., 2014). Premature degradation was reported for another prodrug after incubation in fluids extracted from rabbit intestine (Bali et al., 2015). The recorded degradation can be attributed to the effect of carboxylesterases. These carboxylesterases are known to exist in several organs including the intestine. These enzymes can significantly reduce the bioavailability of substrate drug (Schiel et al., 2007; Laizure et al., 2013). This premature degradation can result in liberation of the parent compound which is less lipophilic compared with the corresponding prodrug with subsequent reduction in the membrane permeability of the drug. This can explain the poor bioavailability of candesartan cilexitil. 
Candesartan cilexetil is suggested to be a substrate for both CES1 and CES2 (Borde et al., 2012; Laizure et al., 2013). This suggestion was based on the recorded degradation of the drug in presence human and dog intestinal fluids with the drug preserving its structure in absence of enzymes (Borde et al., 2012).

Incubation of the drug in the small intestinal fluid in presence of $0.1 \%$ Pluronic F68 did not enhance the stability of candesartan compared with the corresponding pure drug solution. The same trend was obtained in case of colonic fluids (Figure 6).

Incubation of the drug in the intestinal fluids in presence of $0.1 \%$ Tween 80 resulted in significant increase in the stability of the drug compared with the corresponding pure solution. In presence of such concentration of Tween 80 the drug retained about $55 \%$ of its potency compared to $17 \%$ remaining in its absence after 30 minutes. The same trend was recorded after incubation in colonic fluids with the drug retaining $74 \%$ of its potency in the first 30 minutes. These results showed a promising stabilizing effect of Tween 80. Accordingly the efficacy of Tween 80 was tested at higher concentrations. Increasing the concentration of Tween 80 to $0.3 \%$ resulted in further increase in the drug stability with the drug retaining 80 and $85 \%$ of its potency after 30 minutes incubation in small intestinal and colonic fluids, respectively. The drug retained $70.2 \%$ and $69.4 \%$ of its potency in the same fluids after 1 hour and retained $50.4 \%$ and $56 \%$ after 1.5 hours (Figure 6). Further increase in the concentration of Tween 80 to $0.5 \%$ was associated with stability enhancement of candesartan celixitil after incubation in intestinal fluids. The amount drug remaining after 30 minutes was 88.9 and $87.8 \%$ in case of small intestinal and colonic fluids, respectively. The efficacy of Tween 80 was concentration dependent with the stability of the drug increasing at higher concentrations of Tween 80 (Figure 6). This finding may suggest enzyme inhibitory effect of Tween 80 as a mechanism for stabilization of the drug in intestinal fluids. Tween 80 was previously shown to inhibit the carboxlesterase activity (Zhang et al., 2014). Another possible explanation may depend on micelle formation with subsequent entrapment of significant concentrations of the lipophilic drug within the micelles. This can protect the drug from being attacked by the enzyme. In micellar solution there is an equilibrium between the free drug and drug in the micelle with the concentration of the free drug reducing with increasing concentrations of the surfactant. Accordingly, Tween is expected to be more effective as stabilizer at higher concentrations which correlates with the recorded results in the current study. Similar stability enhancement was recorded for ester-based produg in presence of cremophore RH4 which is another micelle forming material (Bali et al., 2015).

\section{CONCLUSION}

Solid dispersion formation of candesartan with PVP, HPMC or Pluronic F68 resulted in significant enhancement in the dissolution rate of the drug. The dissolution enhancement was retained in case of ternary solid dispersion of the drug with PVP with either Pluronic or Tween 80. The enhanced dissolution was mainly due to change in the crystalline structure of the drug after solid dispersion formation. The drug was shown to be subject to premature degradation in the intestinal fluid. This degradation was significantly inhibited in presence of Tween 80 . The stability enhancing effect of Tween 80 is concentration dependent with the surfactant becoming more effective at higher concentration.

\section{REFERENCES}

Akbari J, Saeedi M, Morteza-Semnani K, Kelidari HR, Moghanlou FS, Zareh G, Rostamkalaei S. The Effect of Tween 20, 60, and 80 on Dissolution Behavior of Sprionolactone in Solid Dispersions Prepared by PEG 6000. Adv Pharmac Bulletin, 2015; 5: 435-441.

Bali DE, Osman MA, El Maghraby GM. Enhancement of Dissolution Rate and Intestinal Stability of Clopidogrel Hydrogen Sulfate. Eur J Drug Metab Pharmacokinet, 2015; DOI 10.1007/s13318-015-03114. [In press].

Barakat NS, Elbagory IM, Almurshedi AS. Controlled-Release Carbamazepine Granules and Tablets Comprising Lipophilic and Hydrophilic Matrix Components. AAPS Pharm Sci Tech, 2008; 16: 57-65.

Boghra RJ, Kothawade PC, Belgamwar VS, Nerkar PP, Tekade AR,Surana SJ. Solubility, Dissolution Rate and Bioavailability Enhancement of Irbesartan by Solid Dispersion Technique. Chem Pharm Bull, 2011; 59:438-441.

Borde AS, Karlsson EM, Andersson K, Björhall K, Lennernäs $\mathrm{H}$, Abrahamsson B. Assessment of enzymatic prodrug stability in human, dog and simulated intestinal fluids. Eur J Pharmaceut Biopharmaceut, 2012; 80: 630-637.

Costa P, Lobo JMS. Modeling and comparison of dissolution profiles. European Journal of Pharmaceutical Sciences, 2001;13:123-133.

Detroja C, Chavhan S, Sawant K. Enhanced Antihypertensive Activity of Candesartan Cilexetil Nanosuspension: Formulation, Characterization and Pharmacodynamic Study. Sci Pharm, 2011; 79: 635651.

Devi AS, Peddinti D, Pinnika A. Formulation and Evaluation of Solid Dispersion Tablets of Poorly water soluble drug candesartan cilexetil using poloxamer 407. Int J Pharm Sci Rev Res, 2014; 29: 67-73.

El Maghraby GM, Alomrani AH. Synergistic Enhancement of Itraconazole Dissolution by Ternary System Formation with Pluronic F68 and Hydroxypropylmethylcellulose. Sci Pharm, 2009; 77: 401-417.

El Maghraby GM, Elsergany RN. Fast disintegrating tablets of nisoldipine for intra-oral administration. Pharm Dev Technol, 2014; 19: 641-650.

El-Gizawy SA, Osman MA, Arafa MF, El Maghraby GM. Aerosil as a novel co-crystal co-former for improving the dissolution rate of hydrochlorothiazide. Int J Pharm, 2015; 478: 773-778.

Emami S, Valizadeh H, Islambulchilar Z, Zakeri-Milani P. Development and Physicochemical Characterization of Sirolimus Solid Dispersions Prepared by Solvent Evaporation Method. Adv Pharm Bull, 2014; 4: 369-374.

Fathy M, El-Badry M. Preparation and evaluation of piroxicam - pluronic solid dispersions. Bull Pharm Sci Assiut University, 2003; 26: 97-108.

Gao F, Zhang Z, Bu H, Huang Y, Gao Z, Shen J, Zhao C, Li Y. Nanoemulsion improves the oral absorption of candesartan cilexetil in rats: Performance and mechanism. J Control Rel, 2011; 149: 168-174.

Gorajana A, Rajendran A, Yew LM, Dua K. Preparation and characterization of cefuroxime axetil solid dispersions using hydrophilic carriers. Int J Pharm Investig, 2015; 5: 171-178.

Gurunath S, Nanjwade BK, Patila PA. Enhanced solubility and intestinal absorption of candesartan cilexetil solid dispersions using everted rat intestinal sacs. Saudi Pharm J, 2014; 22: 246-257.

Ha E, Choo G, Baek I, Kim M. Formulation, Characterization, and in Vivo Evaluation of Celecoxib-PVP Solid Dispersion Nanoparticles Using Supercritical Antisolvent Process. Molecules, 2014; 19: $20325-$ 20339. 
Ha E, Kim J, Baek I, Yoo J, Jung Y, Moon HR, Kim M. Development of megestrol acetate solid dispersion nanoparticles for enhanced oral delivery by using a supercritical antisolvent process. Drug Design Development and Therapy, 2015; 9: 4269-4277.

He X, Pei L, Tong HHY, Zheng Y. Comparison of Spray Freeze Drying and the Solvent Evaporation Method for Preparing Solid Dispersions of Baicalein with Pluronic F68 to Improve Dissolution and Oral Bioavailability. AAPS Pharm Sci Tech, 2011; 12: 104-113.

Husain A, Azim MS, Mitra M, Bhasin PS. A Review on Candesartan: Pharmacological and Pharmaceutical Profile. J App Pharm Sci, 2011; 01: 12-17.

Kamalakkannan V, Puratchikody A, Ramanathan L. Development and characterization of controlled release polar lipid microparticles of candesartan cilexetil by solid dispersion. Res Pharm Sci, 2013; 8: 125-136.

Karavas E, Georgarakis E, Sigalas MP, Avgoustakis K, Bikiaris D. Investigation of the release mechanism of a sparingly water-soluble drug from solid dispersions in hydrophilic carriers based on physical state of drug, particle size distribution and drug-polymer interactions. Eur J Pharmaceut Biopharmaceut, 2007; 66: 334-347.

Khan KA, Rhodes CT. Effect of compaction pressure on the dissolution efficiency of some direct compression systems. Pharm Acta Helv, 1972 ;47:594-607.

Khan KA. The concept of dissolution efficiency. J Pharm Pharmacol, 1975; 27, 48-49.

Kura AU, Hussein-Al-Ali SH, Hussein MZ, Fakurazi S. Preparation of Tween 80-Zn/Al-Levodopa-Layered Double Hydroxides Nanocomposite for Drug Delivery System. The Scientific World Journal, 2014; Volume 2014: 10 pages.

Laizure SC, Herring V, Hu Z, Witbrodt K, Parker RB. The role of human carboxylesterases in drug metabolism: have we overlooked their importance?. Pharmacotherapy, 2013; 33: 210-222.

Liu Z, Cui L, Yu D, Zha Z, Chen L. Electrosprayed core-shell solid dispersions of acyclovir fabricated using an epoxycoated concentric spray head. Int J Nanomed, 2014; 9: 1967-1977.

Mar1'n MT, Margarit MV, Salcedo GE. Characterization and solubility study of solid dispersions of flunarizine and polyvinylpyrrolidone. Farmaco, 2002; 57: 723-727.

Mehta MR, Machhaliya RY, Patel CN, Daraji HM. Formulation and evaluation of sublingual tablet of candesartan cilexetil. IJPRBS, 2014; 3: $900-925$

Meka VS, Pillai S, Dharmalingham SR, Sheshala R, Gorajana A. Preparation and in vitro characterization of a non-effervescent floating drug delivery system for poorly soluble drug, glipizide. Acta Poloniae Pharmaceutica ñ Drug Research, 2015; 72: 193-204.

Mesnukul A, Yodkhum K, Phaechamud T. Solid Dispersion Matrix Tablet Comprising Indomethacin-PEG-HPMC Fabricated with Fusion and Mold Technique. Indian J Pharm Sci, 2009; 71: 413-420.

Nekkanti V, Karatgi P, Prabhu R, Pillai R. Solid SelfMicroemulsifying Formulation for Candesartan Cilexetil. Aaps Pharmscitech, 2010; 11: 9-17.

Nekkanti V, Pillai R, Venkateshwarlu V, Harisudhan T. Development and characterization of solid oral dosage form incorporating candesartan nanoparticles. Pharm Dev Technol, 2009; 14: 290-298.
Nishimuta JH, Houston B, Galetin A. Hepatic, Intestinal, Renal, and Plasma Hydrolysis of Prodrugs in Human, Cynomolgus Monkey, Dog, and Rat: Implications for In Vitro-In Vivo Extrapolation of Clearance of Prodrugs. Drug Metab Dispos, 2014; 42: 1522-1531.

Rashid R, Kim DW, Yousaf AM, Mustapha O, Din F, Park JH, Yong CS, Oh Y, Youn YS, Kim JO, Choi H. Comparative study on solid self-nanoemulsifying drug delivery and solid dispersion system for enhanced solubility and bioavailability of ezetimibe. Int J Nanomed, 2014; 10: 6147-6159.

Reddy MS, Goud PS, Apte SS. Solubility enhancement of candesartan cilexetil by self emulsifying drug delivery systems. IJPSR, 2012; 3: 2098-2104.

Schiel M A, Green S, Davis WI, Sanghani PC, Bosron WF, Sanghani SP. Expression and Characterization of a Human Carboxylesterase 2 Splice Variant. J pharmacol Exp Ther, 2007; 323: 94 101.

Sekharan TR, Palanichamy S, Tamilvanan S, Shanmuganathan S, Thirupathi AT. Formulation and Evaluation of Hydroxypropyl Methylcellulose-based Controlled Release Matrix Tablets for Theophylline. Indian J Pharm Sci, 2011; 73: 451-456.

Sezgin-Bayindir Z, Antep MN, Yuksel N. Development and Characterization of Mixed Niosomes for Oral Delivery Using Candesartan Cilexetil as a Model Poorly Water-Soluble Drug. AAPS Pharm. Sci. Tech, 2014; 16: 108-117.

Shaikh SM, Avachat AM. Enhancement of solubility and permeability of Candesartan cilexetil by using different pharmaceutical interventions. Curr Drug Deliv, 2011; 4: 346-353.

Surampalli G, Nanjwade BK, Patil PA. Corroboration of naringin effects on the intestinal absorption and pharmacokinetic behavior of candesartan cilexetil solid dispersions using in-situ rat models. Drug Dev. Ind. Pharm, 2015; 41: 1057-1065.

Yan H, Zhang Z, Jiang Y, Ding D, Sun E, Jia X. An attempt to stabilize tanshinone IIA solid dispersion by the use of ternary systems with nano- $\mathrm{CaCO}_{3}$ and poloxamer 188. Pharmacogn Mag, 2014; 10: S311-S317.

Zhang C, Xu Y, Zhong Q, Li X, Gao P, Feng C, Chu Q, Chen Y., Liu D. In Vitro Evaluation of the Inhibitory Potential of Pharmaceutical Excipients on Human Carboxylesterase 1A and 2. Plos one, 2014; 9: 1-8.

Zhang Z, Gao F, Bu H, Xiao J, Li Y. Solid lipid nanoparticles loading candesartan cilexetil enhance oral bioavailability: in vitro characteristics and absorption mechanism in rats. Nanomed: Nanotech, Biol, and Med, 2012; 8: 740-747.

\section{How to cite this article:}

Fayed ND, Osman MA, El-Maghraby GM. Enhancement of dissolution rate and intestinal stability of candesartan cilexitil. J App Pharm Sci, 2016; 6 (05): 102-111. 